\title{
Failure and energy absorption of plastic and composite chiral honeycombs
}

\author{
A. Airoldi ${ }^{1}$, P. Bettini ${ }^{1}$, M. Zazzarini ${ }^{1}$ \& F. Scarpa ${ }^{2}$ \\ ${ }^{I}$ Dipartimento di Ingegneria Aerospaziale, Politecnico di Milano, Italy \\ ${ }^{2}$ Department of Aerospace Engineering, University of Bristol, UK
}

\begin{abstract}
This paper investigates the crushing response of honeycombs having a chiral geometry, a non centre-symmetric topology made of circular cylinders connected by ligaments. Buckling and post-buckling responses of flatwise compressed plastic chiral honeycombs are numerically investigated. Results indicate that plastic chiral honeycombs can carry increasing loads beyond local buckling of ligaments, though the post-buckling response does not lead to high energy absorption performances. The characteristics of chiral topology can be exploited by adopting composite materials. The composite units produced by using a specific technological process presented in the paper and endowed with a bevel trigger failed by progressive crushing and obtained promising specific energy absorption levels.
\end{abstract}

Keywords: honeycombs, energy absorption, chiral structures, composites.

\section{Introduction}

The design of impact tolerant and crashworthy structures is one of the motivations for the development of honeycombs with high crushing strength and energy absorption performances. Honeycombs are typically used as lightweight and structurally efficient core material in composite sandwich structures, with the role of carrying the applied normal loads and the transverse shear internal forces. In such a context, the crushing strength and the energy absorption properties of the core influence the damage levels experienced by sandwich elements in low-energy impacts [1]. Moreover, honeycombs are also used in the design of efficient energy absorbers for various applications. In particular, honeycomb is often adopted as filling material inside light alloy crushing tubes, such as in the absorber for racing cars, presented by Manusamy and Barton [2] or 
in the studies presented by Zhang et al. [3]. These types of absorbers exploit the typical axial collapse mode of honeycombs, which is characterized by an almost constant crushing load level.

Alternatives to classic hexagonal honeycombs were studied by several researchers. Honeycombs with a negative in-plane Poisson's coefficient (auxetic) were developed to reduce problems related to the production of doubly curved sandwich panels [4]. Auxetic honeycombs can also be produced based on chiral geometry, a special non centre-symmetric topology that was originally proposed by Lakes [5]. Chiral topologies consist of circular elements, called nodes, connected by straight ligaments. The ligaments are tangential to the nodes and tend to wind or unwind about the nodes under the action of a compressive or tensile in-plane force, respectively. Such deformation mechanism originates a negative Poisson's coefficient and has been exploited to design morphing structures that can smoothly change their shape under the actions of aerodynamic loads [6-8].

Although the peculiar auxetic behaviour is obtained for in-plane load conditions, chiral honeycombs also exhibit remarkable out-of-plane properties. Numerical evaluations of buckling loads in flatwise compression of chiral honeycombs are presented by Spadoni et al. [9]. Results shows that chiral honeycombs present a superior design flexibility with respect to classic hexagonal configurations and higher buckling loads for the same relative densities. The analytical and numerical linear-elastic models presented by Scarpa et al. [10] obtained similar results, which were experimentally validated by testing plastic chiral honeycombs produced with use of rapid prototyping techniques. Further studies on buckling and a comprehensive discussion on elastic out-of-plane properties in chiral honeycombs are presented in [11] and [12], respectively.

According to the published results, chiral geometries could be considered promising candidates for highly efficient honeycomb cores and energy absorbing systems. However, possible applications must consider the difficulties involved in manufacturing process. Rapid prototyping techniques and machining of solid metallic plates were used to produce plastic and metallic chiral honeycombs [7, 10-12]. A technological process was developed by Bettini et al. [13] for manufacturing chiral honeycombs by using long-fibre reinforced composite materials.

Chiral honeycombs made of carbon/epoxy laminates have been considered for the design of morphing structures $[8,13]$, but they should also present high out-of-plane mechanical properties and could have a great potential of energy absorption. Indeed, it is well known that the activation of progressive crushing in the walls of axially-loaded composite elements provides a crushing response at constant load levels with specific values of absorbed energy that greatly exceed the performances of metallic absorbers [14-17]. Hence, it can be suggested that the inherent buckling strength of chiral topologies could help the activation of the high energy absorption failure modes of composite walls.

The investigation of failure modes and energy absorption in plastic and composite chiral honeycombs are the objectives of this paper. The features of 
chiral topologies are presented and linear buckling analyses of chiral honeycombs are performed and compared with the results of Spadoni et al. [9]. The models of the experiments presented by Scarpa et al. [10] are developed by using an explicit FE approach to better investigate the failure modes and to validate a numerical approach for an accurate prediction of failure of plastic honeycombs. Finally, the technology for the production of composite chiral honeycombs is applied to the production of chiral units that are subjected to crushing experiments for the evaluation of their energy absorbing capabilities.

\section{Flatwise linear buckling of chiral honeycombs}

The chiral unit presented in Fig. 1(A) is representative of the hexachiral topology that will be considered throughout this work. The ligament length, $L$, the distance between the centres of the nodes, $R$, and the node radius, $r$, can be considered the main geometrical parameters. The angle $\vartheta=30^{\circ}$ defines the hexachiral configurations, whereas the angle $\varphi$ can be obtained from the other parameters as indicated in Fig. 1.

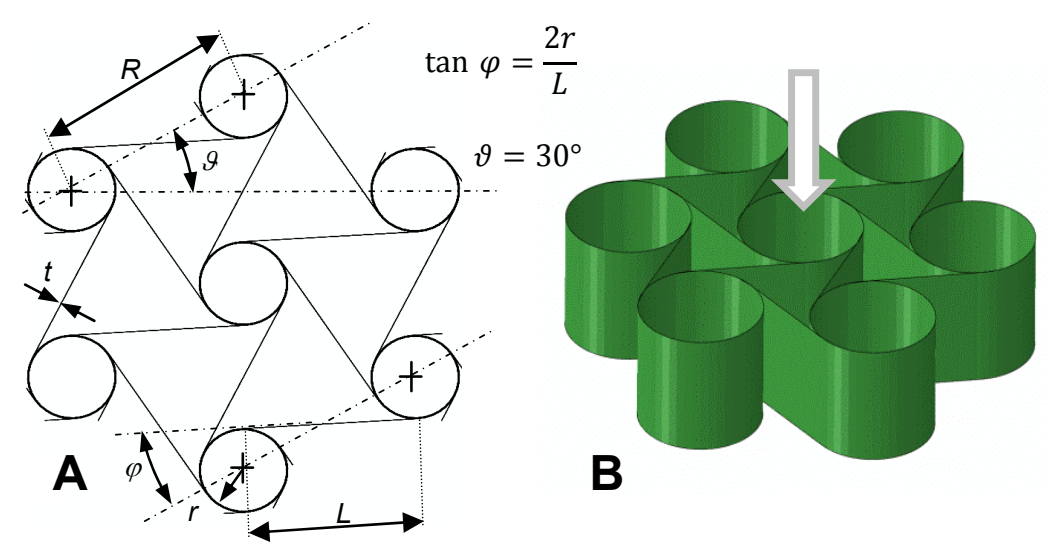

Figure 1: Hexachiral topology with main geometrical parameters (A) and flatwise compression of a chiral unit (B).

The failure of the chiral unit in flatwise compression conditions, which are sketched in Fig. 1(B), can be driven by instability phenomena occurring in the ligaments. Such elements can be considered as panels laterally supported by cylinders, or in the nodes, which can be idealized as cylinders stiffened by the panels. For such a reason the crushing strength of a chiral honeycomb is expected to depend on the ratio $L / R$, or alternatively on the parameter $\alpha=L / r$. As $L / r$ increases, or $L / R$ approaches the unit value, configurations with long ligaments and small nodes are obtained. Preliminary considerations by Spadoni et al. [9] indicate that panel buckling should occur before cylinder buckling for reasonable values of geometrical parameters. Buckling loads of ligaments can be 
predicted by analytical formulations and by means of FE linear buckling analyses of a representative chiral cell.

A FE model of the representative cell proposed in [9] was also developed in the present work. The cell is obtained from the chiral unit of Fig. 1 by cutting half of the external cylinders, as it is shown in Fig. 2(A). Periodic boundary conditions are imposed to the cut edges.

A

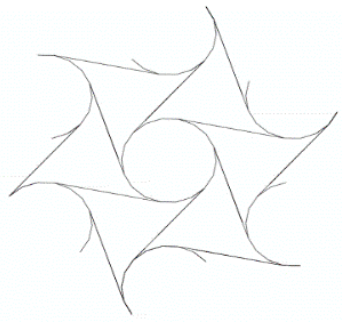

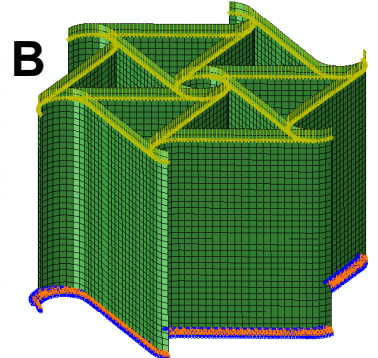

Figure 2: $\quad$ Representative chiral cell (A) and FE model (B).

The FE model was automatically generated by a Matlab ${ }^{\circledR}$ script. A cell model is presented in Fig. 2(B). Nodes at the top end of the cell were left unconstrained and a uniform load distribution with a unit resultant was applied to edge of the shell elements. Nodes at the lower end were fully constrained. Accordingly, boundary conditions and loads were applied as in [10], though a more a refined mesh of 8-noded second order elements was used in this work. A linear buckling analysis was performed by using Abaqus/Standard code. Solution procedure is based on eigenvalue analysis to find the load at the bifurcation of the equilibrium and the associated eigenvectors, which represent the buckling modes. Lanczos method was selected to solve the eigenvalue problem [18].

Different configurations of chiral honeycombs were considered in the range $R / L=0.75 \div 0.80$. The cylinder radius and the wall thickness were set to $16 \mathrm{~mm}$ and $0.2 \mathrm{~mm}$, respectively. A material with $E=2200 \mathrm{MPa}$ and $\nu=0.33$ was used for a direct comparison with data reported [10]. A low Young modulus was selected to consider possible applications to chiral samples produced by using rapid prototyping techniques. Indeed, manufacturing of chiral honeycombs with relatively low wall thickness is not easy and rapid prototyping represents an appealing and feasible solution, though it involves adoption of material with low stiffness and strength value [10-12].

The first buckling modes in the analyses turn out to be associated with the buckling of the panels. Following the definitions given in [9], local modes and global modes can be distinguished as in the examples provided in Fig. 3. In Fig. 4(A), the lowest eigenvalues corresponding to global modes are compared with the numerical results and the analytical predictions presented in [9]. The equation suggested for analytical predictions is also reported in Fig. 4(A). It represents the critical load for each ligaments of the cell and requires a constant $K$, which was set to 5.2 in [9] after a comparison with numerical results. The results confirm that the global buckling of the ligaments can be predicted by 

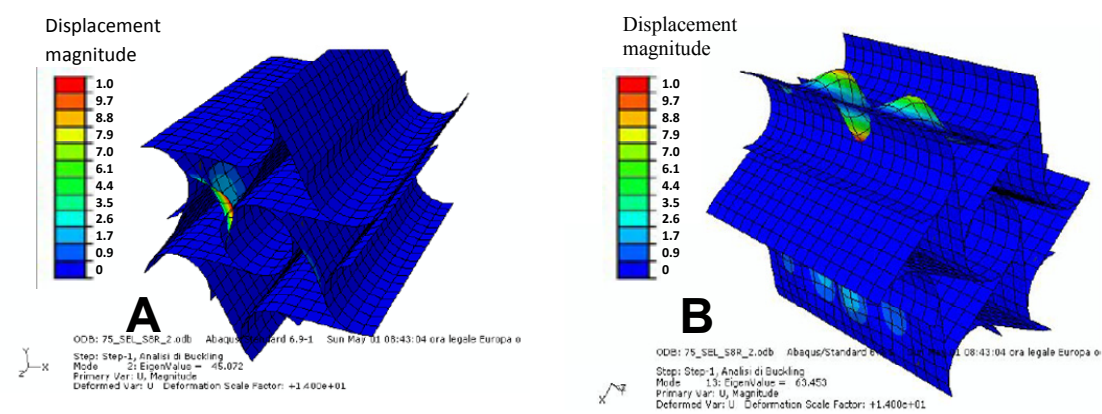

Figure 3: $\quad$ Local (A) and global (B) buckling of ligaments.

considering the buckling of a supported panel and that, as the $L / R$ ratio increases, ligaments become longer and the buckling stress for the cell diminishes. However, the honeycomb relative density also varies with $L / R$ ratio and it can be proved that the performances of chiral honeycomb in terms of compression strength is higher than that of classic hexagonal honeycombs, at the same levels of relative densities [9].

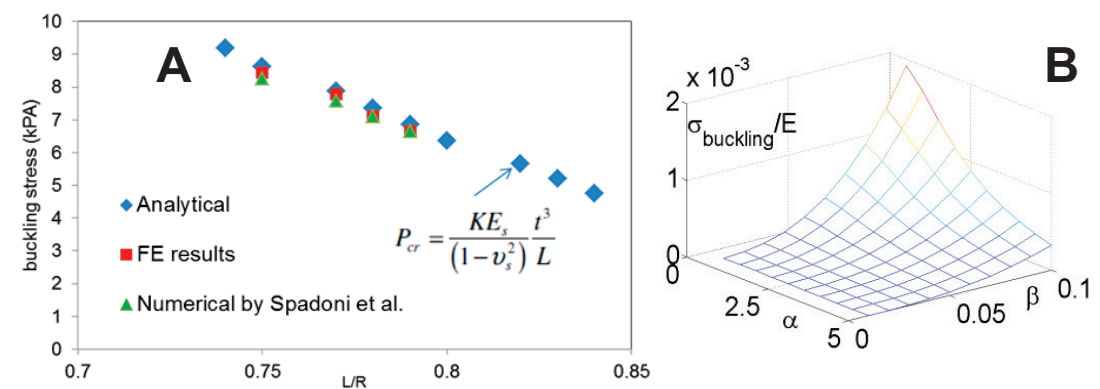

Figure 4: Analytical and numerical buckling stress for different $L / R$ values (A) and buckling stress according to eqn. 1 (B).

In the previous analysis it is assumed that global ligament buckling sets a limit to the load carrying capability of chiral honeycombs. However, the possibility that the honeycomb can carry higher load levels until the failure of cylinder has to be taken into consideration. In the studies by Scarpa et al. [10], failure was investigated by means of linear buckling analyses and by considering a fundamental buckling mode, which also involves the deformation of cylinders. An analytical formulation was developed focusing on cylinder buckling. In the proposed formulation, buckling stress was expressed as a function of material properties, $E$ and $v$, and of two geometrical parameters, $\alpha=L / r$ and $\beta=t / r$ :

$$
\frac{\sigma_{b}}{E}=\frac{\pi}{\sqrt{3\left(1-v^{2}\right)}} \frac{4 \beta^{2}}{8 \pi+3 \sqrt{3} \alpha^{2}}\left(K_{\beta} \beta^{0.8}+K_{\alpha} \alpha\right)
$$


where $K_{\beta}$ and $K_{\alpha}$ are two constants that are set to 7.2 and 0.08 , respectively. Such values were obtained in [10] by fitting the predictions of eqn. 1 with the eigenvalues corresponding to the fundamental buckling modes of a large number of numerical FE analyses. Numerical and analytical predictions were confirmed by the experimental results obtained on PA and ABS chiral honeycombs. The ratio between buckling stress and material Young's modulus provided by eqn. 1 is plotted against parameters $\alpha$ and $\beta$ in Fig. 4(B). Both parameters considerably influence the buckling strength, thus evidencing the significant design flexibility of chiral honeycombs.

The studies by Scarpa et al. [10] highlight the mutual interaction of ligaments and cylinder during the failure of flatwise compressed chiral honeycombs: cylinders support ligaments, but buckling of ligaments influences the cylinder collapse. The predictions provided by the validated semi-analytical formulation confirm that buckling strength of chiral honeycombs is considerably higher than that of hexagonal honeycombs for the same relative density.

\section{Analyses of buckling and post-buckling behaviour of plastic chiral honeycombs}

The development a complete non-linear model for the flatwise compression of chiral honeycombs is presented in this section. The scopes of the numerical activity are a further investigation of the collapse mode in plastic chiral honeycombs and the development of a tool for the direct prediction of buckling and post-buckling response.

The benchmarks for the application of the approach are two test cases presented by Scarpa et al. [10]. The tests were performed on an identical chiral topology having $r=4.5 \mathrm{~mm}, L=20 \mathrm{~mm}$ and $t=1 \mathrm{~mm}$. A first type of honeycomb sample was produced by means of HP nylon powder stereolytography and a second type was made of ABS polymer and manufactured with use of fusion deposition moulding. The honeycomb cores, with a thickness of $20 \mathrm{~mm}$ and in-plane dimensions of $105 \mathrm{~mm} \times 100 \mathrm{~mm}$, were subjected to quasi-static compressive tests.

Two type of models were developed and solved by using Abaqus/Explicit for the analysis of the tests. The first model represents the FE scheme of a chiral honeycomb with dimensions equal to half of the tested specimens. The scheme is presented in Fig. 5(A); it includes 15 cylinders and consists of about 50000 linear shell elements with reduced integration scheme. Boundary conditions were imposed by means of two rigid planes that were set in contact with the FE scheme of the chiral honeycomb. A velocity boundary condition was applied to the upper plane, which is not visible in Fig. 5(A), to model a quasi-static compression test. All the contacts between the ligaments and the cylinder walls were also implemented in the models. The second type of model is based on the reduced FE scheme that is presented in Fig. 5(B). In such a model, the effects of the surrounding cells were represented by means of a set of symmetry boundary conditions, at the edge of the ligaments, as indicated in Fig. 5(C). 


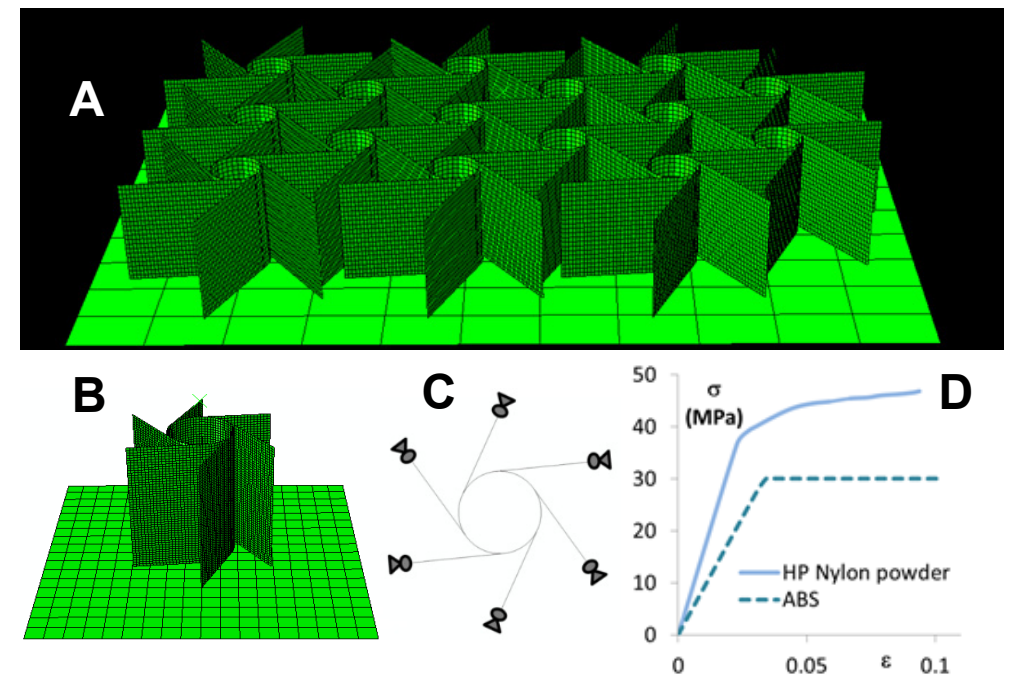

Figure 5: Chiral honeycomb FE model (A), reduced model (B) with boundary conditions (C) and material stress-strain curves (D) for non-linear compression analyses.

Materials were modelled by means of an elastic-plastic constitutive law with a Von Mises associated flow rule. Following the data provided in [10], the Young's modulus of ABS plastic and HP nylon powder were set to $900 \mathrm{MPa}$ and $1600 \mathrm{MPa}$, respectively. The stress-strain curves used in the model are presented in Fig. 5(D).

The numerical-experimental correlation of the force vs. displacement curves referred to the load carried by a single cylinder are reported in Fig. 6.

The differences between numerical and experimental slopes of the flatwise compressive stress-strain curves can be ascribed to different factors.
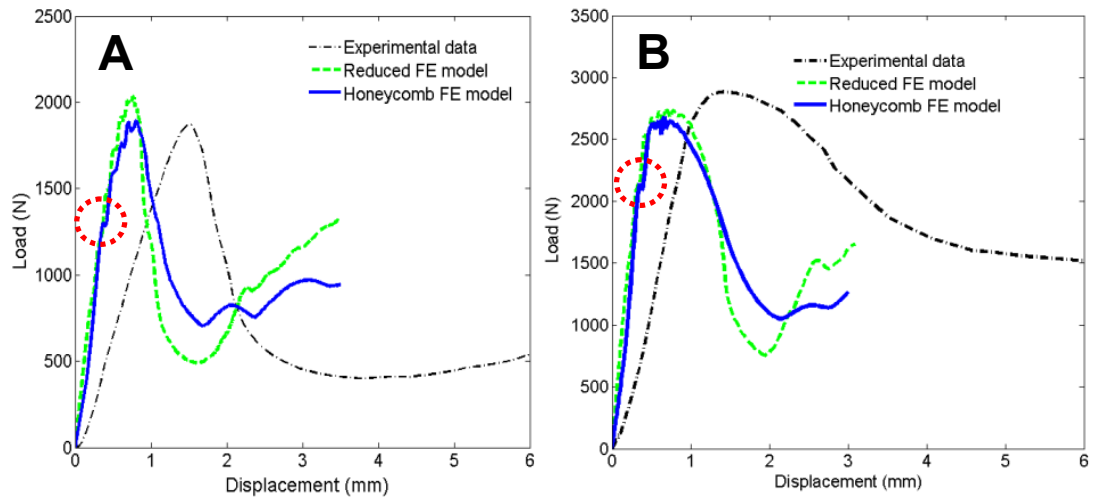

Figure 6: Numerical-experimental correlation for ABS (A) and HP nylon powder (B) chiral honeycombs. 
An important factor is related to the effective morphology of the core material obtained in the manufacturing process. The ABS plastic sample has effectively not a single ligament, but two ligaments in contact through incomplete fusion. Therefore, the equivalent stiffness of the material is decreased by an effective porosity existing within the ligament, and it goes to affect the overall compressive stiffness of the honeycombs. The HP nylon powder sample has also a distributed porosity within the ligaments and cylinders of the hexachiral configuration. Porosity is formed when the powders receive low levels of energy density (ED), typically between $9 \mathrm{~W}$ and $11 \mathrm{~W}$, which was the case for the sample produced [19]. Porosity decreased the stiffness of the material compared to a pristine configuration, and therefore affects also the compressive modulus of the honeycomb.

Moreover, it is worth noting that tests were performed by using different fixtures [10]. Failures in the ABS samples were usually observed at one angle of the honeycomb unless great care was used to position the specimens, though fixture was endowed with self-aligning systems. The tests of HP nylon powder samples were performed by using two steel plates that were manually moved until a visible closure of all gaps between plate surfaces and honeycomb sample. Such alignment problems could have been originated by geometric imperfections in the samples produced by rapid prototyping technique, which could have influenced the results.

The sequence shown in Fig. 7 is taken from the analysis of the HP nylon powder test and illustrates the collapse mode obtained in the model. Fig. 7(A) is referred to the initial part of the analysis, whereas Fig. 7(B) is taken after a first small load oscillation, which is denoted in Fig. 6 by a dotted circle. Beyond such load level, buckles appear on the ligaments. Fig. 7(C) is taken at the maximum load where buckles are developing at one end of the nodes. Considering the stress-strain curve shown in Fig. 5(D), it is observed that Von Mises stress at maximum load are higher than the yield stress of the HP nylon powder material model. Hence, according to numerical results, the collapse is likely to be influenced by material yielding and inelastic behavior. The effects of material porosity due to technological process on the inelastic response of the core material can be considered to explain the differences in the amplitude of the load peaks. Finally, Fig. 7(D) is representative of the post-buckling behaviour.

Despite the difficulties related to peculiarities of the materials used in manufacturing, maximum loads are captured by numerical analyses and the collapse modes are in good agreement with the experimental evidences presented in [10].

Overall, the analysis of the data indicates that the chiral honeycomb in the considered configuration can withstand to load increments beyond the onset of ligament buckling. Final failure occurs with the development of local buckles in the nodes. Moreover, the effects of ligaments and the contacts between the cylinder and the buckled ligaments promotes an asymmetric buckling model in the nodes (Fig. 7(D))

Hence, the stiffening effects of cylinders and the influence of ligament buckling on the cylinder collapse, discussed in [10], are confirmed by the 


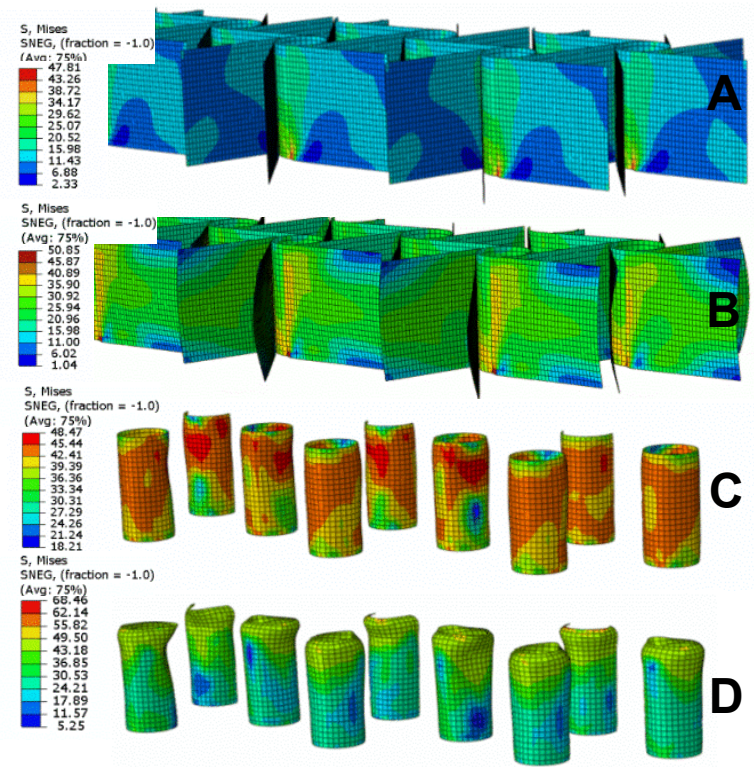

Figure 7: Collapse mode in the numerical honeycomb model.

developed numerical non-linear approach. However, the post-buckling response obtained in the tests presented is not appropriate for enhanced energy absorption performances. Indeed, in the configuration considered, collapse is not characterised by a constant crushing load and load levels in the post-buckling response are considerably lower than initial peaks. According to the results, the computational approach based on the reduced numerical model can be considered an efficient computational tool for the optimization of energy absorption performance in plastic chiral honeycombs.

\section{Production and crushing tests of composite chiral honeycombs}

Absorbers based on chiral topologies can be also developed by exploiting the technological process developed by Bettini et al. for manufacturing chiral honeycombs made of carbon/composite laminates [13]. The process was originally developed for relatively large chiral units (with $L=75 \mathrm{~mm}$ and $r=18$ $\mathrm{mm}$ ) and the application to a hexachiral configuration with shorter ligaments and smaller nodes $(L=19.3 \mathrm{~mm}$ and $r=10.3)$ is herein presented.

The production process consists of two steps: the production of ligaments (Figs. 8(A), (B)) by means of a conventional vacuum bag technology and the assembly and bonding of the chiral unit (Figs. 8(C), (D), (E)). Nodes are formed by the superposition of the ligament curved ends, which are bonded together in the second step of the process. The assembly mould prepared for the production of composite chiral units, shown in Fig. 8(C), consists of an array of machined 

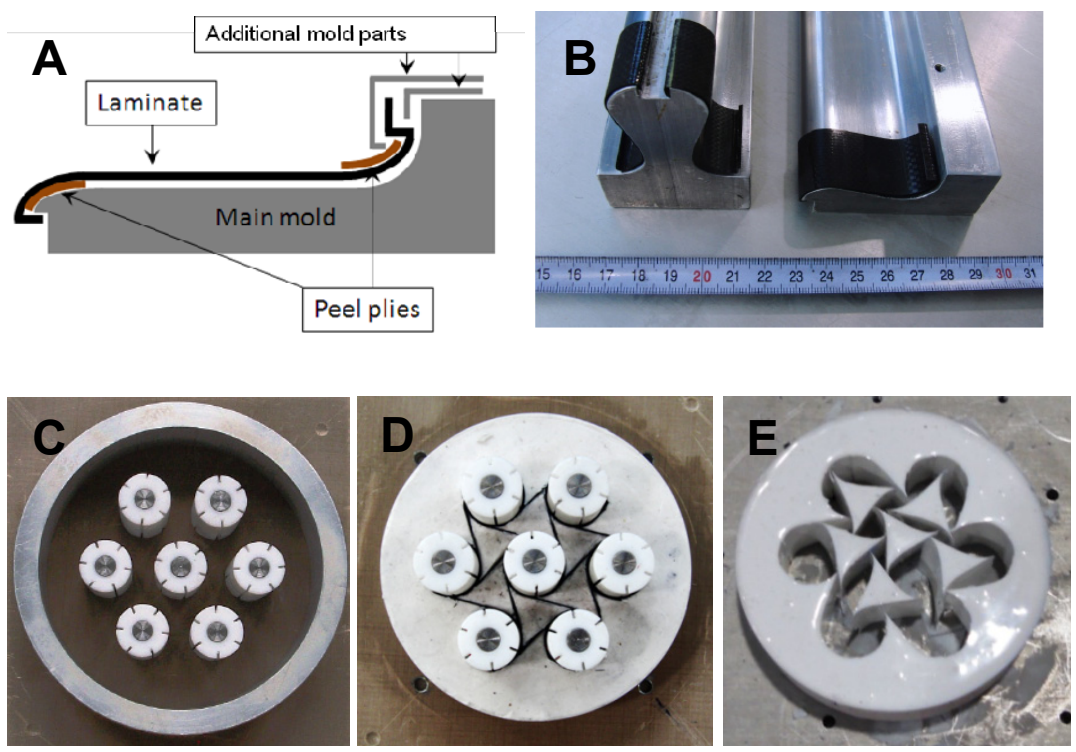

Figure 8: Manufacturing of composite chiral units: production of ligaments (A, B), assembly of chiral unit (C), assembled unit (D) and silicon rubber inserts $(\mathrm{E})$.

PTFE cylinders within a circular dam. Ligaments were positioned around the PTFE cylinders after the application of strips of adhesive film on the curved ends (Fig. 8(D)). Silicon rubber inserts, shown in Fig. 8(E) were preliminarily produced by using the same mould and then introduced in the gaps of the assembly. Then, the assembly was inserted into a vacuum bag and adhesive is cured by applying a pressure-temperature cycle. The rubber inserts have a considerable CTE coefficient and allow the application of the pressure on the adherends during curing [13]. A chiral unit sample is shown in Fig. 9(A).

Four $24 \mathrm{~mm}$-thick chiral units were produced and tested with two types of lamination sequences, made of 4 and 8 plies, respectively. The pre-preg used in the lamination is a plain weave fabric SEAL CC90/ET445 with a cured ply thickness of $0.1 \mathrm{~mm}$. One specimen for each type of lamination sequence was machined along the top edge of the walls to produce a $45^{\circ}$ bevel. The bevel was introduced to trigger the activation of progressive crushing mode in the walls of the honeycomb structures. Quasi-static compression tests were conducted by using an MTS/810 static test system and the fixture presented in Fig. 9(B). Chiral units were positioned on a lower plate and compressed by a circular steel plate connected by a spherical joint to the upper cross-head of the test system. Tests were performed at a displacement rate of $0.25 \mathrm{~mm} / \mathrm{min}$. Table 1 summarizes the characteristics of the samples and reports for each test the maximum load, the load uniformity ratio (LUR), which represents the ratio of average to maximum 

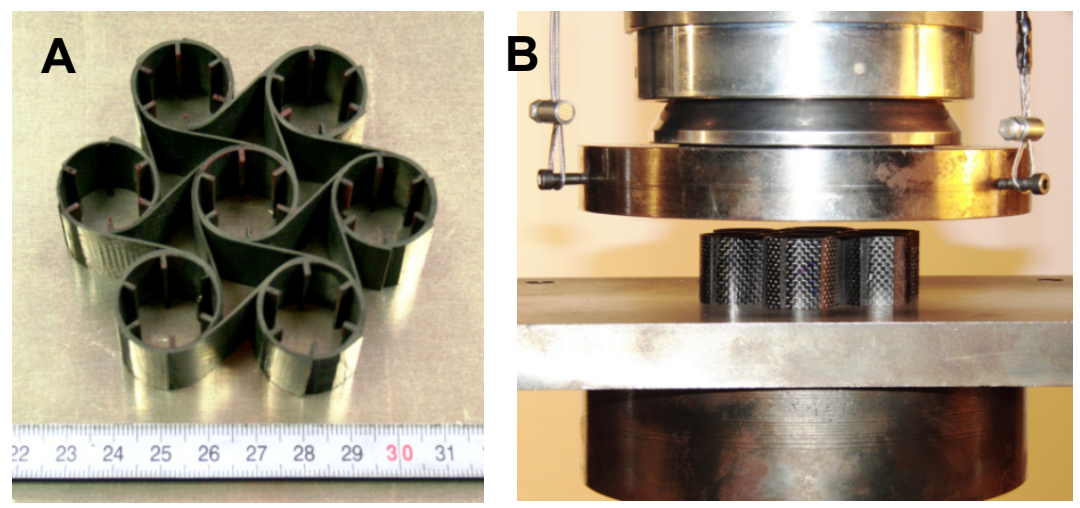

Figure 9: $\quad$ Composite chiral unit (A) and test set-up (B).

Table 1: $\quad$ Tests performed on composite chiral honeycombs.

\begin{tabular}{|c|c|c|c|c|c|c|}
\hline$\#$ & Lay-up & $\begin{array}{c}\text { thickness } \\
(\mathrm{mm})\end{array}$ & $\begin{array}{c}45^{\circ} \\
\text { bevel }\end{array}$ & $\begin{array}{c}\text { Max Load } \\
(\mathrm{kN})\end{array}$ & LUR & $\begin{array}{c}\text { SEA } \\
(\mathrm{kJ} / \mathrm{kg})\end{array}$ \\
\hline 1 & {$[0 / 45 /-45 / 0]_{\mathrm{S}}$} & 0.8 & No & 174 & - & - \\
\hline 2 & {$[0 / 45 / 0 /-45]$} & 0.4 & No & 42 & 0.22 & 22.6 \\
\hline 3 & {$[0 / 45 /-45 / 0]_{\mathrm{S}}$} & 0.8 & Yes & 85 & 0.89 & 96.5 \\
\hline 4 & {$[0 / 45 / 0 /-45]$} & 0.4 & Yes & 29 & 0.55 & 38.7 \\
\hline
\end{tabular}

load, and the absorbed energy per unit of crushed mass (specific absorbed energy: SEA).

The force vs. displacements responses obtained in the tests are reported in Fig. 10, whereas the failure modes of the two samples with trigger are shown in Fig. 11. The chiral unit \#1 failed by brittle failure at a load of $174 \mathrm{kN}$, but the same configuration featured with the bevel (unit \#3) obtained a remarkable energy absorption performance. The force vs. displacement curve indicates almost constant crushing loads with an LUR of 0.89 . SEA is almost $100 \mathrm{~kJ} / \mathrm{kg}$. In the failure mode shown in Fig. 11(A), the activation of a progressive crushing in all nodes and ligaments of the units is apparent

The failure of the $0.4 \mathrm{~mm}$-thick unit without bevel was characterised by a peak at $42 \mathrm{kN}$, which was followed by buckling and debonding of ligaments. In the sample with trigger, buckling occurred on the external nodes of the unit but progressive crushing was activated in the internal walls, as it shown in Fig. 11(B). A LUR of 0.55 and a SEA of $38.7 \mathrm{~kJ} / \mathrm{kg}$ were obtained.

The presented performance can be compared with some results presented in literature. According to the review presented in [15], a SEA level of $100 \mathrm{~kJ} / \mathrm{kg}$ is obtained in carbon/epoxy laminated only by using [0/ \pm 15$]$ lay-up, whereas the maximum value reported for [0/ \pm 45$]$ laminates is $65 \mathrm{~kJ} / \mathrm{kg}$. More recently, examples of SEA values exceeding $100 \mathrm{~kJ} / \mathrm{kg}$ are reported, such as in $[16,17]$ with maximum levels of $116 \mathrm{~kJ} / \mathrm{kg}$. Although the specific energy absorption 

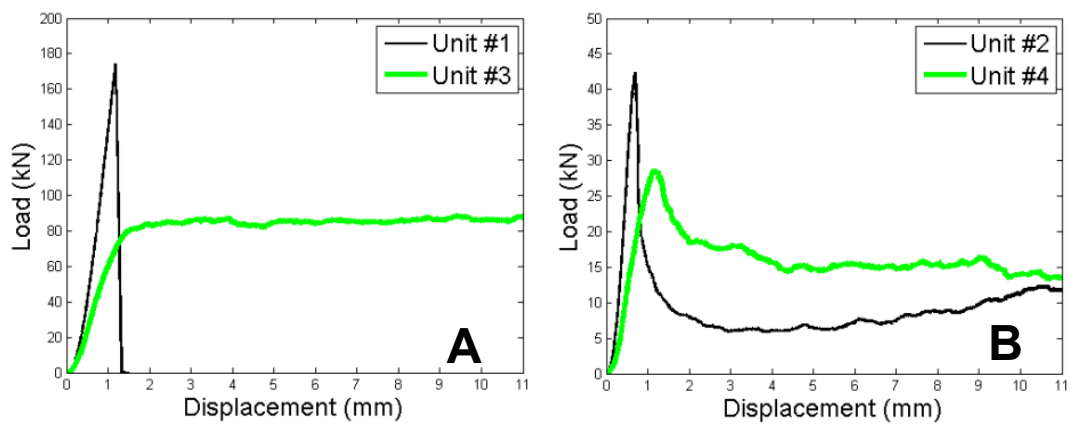

Figure 10: Force vs. displacement curves for thick (A) and thin (B) composite chiral units.
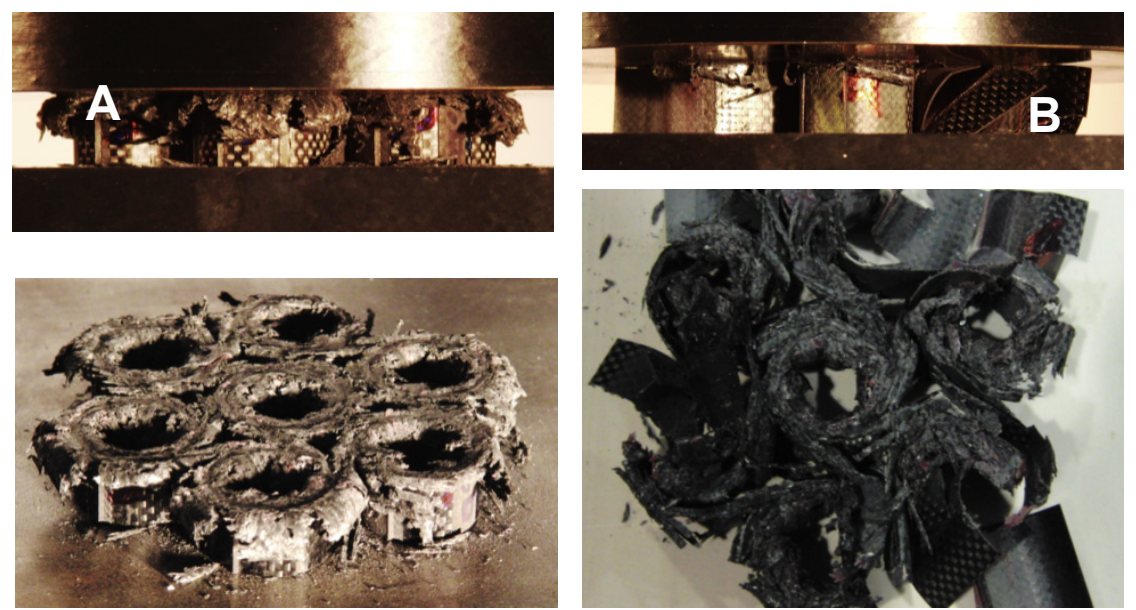

Figure 11: $\quad$ Failure modes of unit \#3 (A) and \#4 (B).

levels largely depend on the specific material properties, it can be observed that the quantitative performance obtained by the thick composite unit is noticeable. The activation of progressive crushing in the central cylinder of the thin composite unit has also to be remarked. Activation of progressive crushing in very thin walls indicates that the design flexibility of chiral topology presented in Fig. 4(B) can be actually exploited to design composite chiral honeycombs with high energy absorption efficiency for a very wide range of crushing pressures.

\section{Concluding remarks}

The presented activities confirm that chiral topologies present considerable high out-of-plane compressive strength and a potential for the development of highly efficient energy absorbers. The collapse of plastic chiral honeycombs has been analysed by means of explicit FE computation and the mutual interactions 
between nodes and cylinders in the compressive response have been highlighted. A numerical approach has been validated and could represent an efficient tool to optimize energy absorption performances of honeycombs made of ductile materials. Moreover, a process for manufacturing of composite chiral units has been presented and a set of carbon composite samples has been subjected to quasi-static crushing tests. In the units endowed with a trigger, progressive crushing of composite walls has been activated even in thin carbon composite walls $(0.4 \mathrm{~mm})$ and a specific absorbed energy of $100 \mathrm{~kJ} / \mathrm{kg}$ has been recorded for units with a wall thickness less than $1 \mathrm{~mm}$. Such results indicates that the inherent buckling strength of chiral topologies can be effectively exploited in composite chiral units, which can be considered very promising for the development of highly efficient energy absorbing structures.

\section{References}

[1] Abrate S., Impact on composite structures, CUP, 1998.

[2] Munusamy R., Barton D.C, Lightweight impact crash attenuators for a small Formula SAE race car, International Journal of Crashworthiness. 15(2), pp. 223-234, 2010.

[3] Zhang Z., Liu S., Tang Z., Comparison of honeycomb sandwich and foamfilled cylindrical columns under axial crushing loads, Thin-Walled Structures 49 pp. 1071-1079, 2011.

[4] Evans KE. Design of doubly curved sandwich panels with honeycomb cores, Computer Structures 17, pp. 95-111, 1991.

[5] Lakes R.S., Deformation mechanisms of negative Poisson's ratio materials: structural aspects, Journal of Materials Science 26, pp. 2287-2292, 1991.

[6] Bornengo, D., Scarpa, F., and Remillat, C., Evaluation of Hexagonal Chiral Structure for Morphing Airfoil Concept, Journal of Aerospace Engineering 219, pp. 185-192, 2005.

[7] Spadoni, A., Ruzzene, M., Numerical and Experimental Analysis of The Static Compliance of Chiral Truss-Core Airfoils, Journal of Mechanics of Materials and Structure 2(5), pp. 965_981, 2007.

[8] Airoldi A., Crespi C., Quaranta G., Sala G., Design of a morphing airfoil with composite chiral structure, Journal of Aircraft, In press, DOI: 10.2514/1.C031486.

[9] Spadoni A., Ruzzene M., Scarpa F., Global and Local Linear Buckling Of Chiral Cellular Structure, Physica Status Solidi B 242(3), pp. 695-709, 2005.

[10] Scarpa F., Blain S., Lew T., Perrott D., Ruzzene M., Yates J.R., Elastic buckling of hexagonal chiral cell honeycombs, Composites Part A 38, pp. 280-289, 2007.

[11] Lorato A., Innocenti P., Scarpa F., Alderson A., Alderson K.L., Zied K.M., Ravirala N., Miller W., Smith C.W., Evans K.E., The transverse elastic properties of chiral honeycombs, Composite Science and Technology 70, pp. 1057-1063, 2010. 
[12] Miller W., Smith C.W., Scarpa F., Evans K.E., Flatwise buckling optimization of hexachiral and tetrachiral honeycombs, Composite Science and Technology 70, pp. 1049-1056, 2010.

[13] Bettini P., Airoldi A., Sala G., Di Landro L., Ruzzene M., Spadoni A., Composite chiral structures for morphing airfoils: Numerical analyses and development of a manufacturing process, Composites Part B 41, pp. 133147, 2010.

[14] Kindervater C. M., Georgi H., Composite Strength and Energy Absorption, in Structural Crashworthiness and Failure, Jones N. and Wierzbicki T. editors, Elsevier Applied Science, 1993.

[15] Mamalis, A.G., Robinson, M., Manolakos, D.E., Demosthenous, G.A., Ionnidis, M.B., Carruthers, J., Crashworthy Capability of Composite Material Structures, Composite Structures 37, pp. 109-134, 1997.

[16] Cachi-Savona S., Zang C., Hogg. P., Optimisation of crush energy absorption of non-crimp fabric laminates by through-thickness stitching, Composite Part A 42, pp. 712-722, 2010

[17] Mahdi E., Hamouda A.M.S., Sahari B.B., Khalid Y.A., Experimental quasistatic axial crushing of cone-tube-cone composite systems, Composite Part B 34, pp. 285-302, 2003.

[18] Abaqus Theory Manual, version 6.7, Dassault System, 2007.

[19] Caulfield B., McHugh P.E., Lohfeld S., Dependence of mechanical properties of polyamide components on build parameters in the SLS process, Journal of Materials Processing Technology 182, pp. 477-488, 2010. 